\title{
Free-volume dependent pressure sensitivity of Zr-based bulk metallic glass
}

\author{
Alban Dubach \\ Laboratory of Metal Physics and Technology, Department of Materials, ETH Zurich, 8093 Zurich, \\ Switzerland; and EMPA Materials Science and Technology, 3602 Thun, Switzerland \\ K. Eswar Prasad \\ Department of Materials Engineering, Indian Institute of Science, Bangalore-560012, India \\ Rejin Raghavan \\ EMPA Materials Science and Technology, 3602 Thun, Switzerland; and Department of Materials \\ Engineering, Indian Institute of Science, Bangalore-560012, India \\ Jörg F. Löffler \\ Laboratory of Metal Physics and Technology, Department of Materials, ETH Zurich, \\ 8093 Zurich, Switzerland \\ Johann Michler \\ EMPA Materials Science and Technology, 3602 Thun, Switzerland \\ Upadrasta Ramamurty ${ }^{\text {a) }}$ \\ Department of Materials Engineering, Indian Institute of Science, Bangalore-560012, India
}

(Received 5 March 2009; accepted 4 May 2009)

\begin{abstract}
Instrumented indentation experiments on a Zr-based bulk metallic glass (BMG) in as-cast, shot-peened and structurally relaxed conditions were conducted to examine the dependence of plastic deformation on its structural state. Results show significant differences in hardness, $H$, with structural relaxation increasing it and shot peening markedly reducing it, and slightly changed morphology of shear bands around the indents. This is in contrast to uniaxial compressive yield strength, $\sigma_{y}$, which remains invariant with the change in the structural state of the alloys investigated. The plastic constraint factor, $C=H / \sigma_{\mathrm{y}}$, of the relaxed BMG increases compared with that of the as-cast glass, indicating enhanced pressure sensitivity upon annealing. In contrast, $C$ of the shot-peened layer was found to be similar to that observed in crystalline metals, indicating that severe plastic deformation could eliminate pressure sensitivity.

Microscopic origins for this result, in terms of shear transformation zones and free volume, are discussed.
\end{abstract}

\section{INTRODUCTION}

Experimental assessment of plasticity in amorphous alloys is relatively complicated, compared with that of crystalline metals and alloys. This difference is because of the inhomogeneous nature of deformation in metallic glasses through shear banding as well as the mechanical instability associated with the strain softening postyielding. Pressure sensitivity of plastic flow complicates the matter further, imparting tension/compression asymmetry. In uniaxial tension, fracture ensues almost immediately after yield due to the mechanical instability. Hence, only the yield strength can be measured reliably by tension tests. Flexure loading, which is popular for testing ribbon forms of rapidly quenched glasses, ${ }^{1,2}$ is

\footnotetext{
a) Address all correspondence to this author.

e-mail: ramu@materials.iisc.ernet.in

DOI: 10.1557/JMR.2009.0304
}

not suitable for thicker plates of bulk metallic glasses (BMGs). This is because shear bands become shear cracks, once they are allowed to traverse certain minimum distance, and the large elastic strain energy stored in the specimen gets released upon the initiation of shear cracks, leading to immediate fracture. ${ }^{1}$ In addition, accurate determination of the stress state under flexure loading is complicated by the tension-compression asymmetry that amorphous alloys exhibit. Therefore, uniaxial compression and indentation have been widely used testing techniques for characterizing plasticity in BMGs.

In a previous study, we have critically examined the utility of uniaxial compression testing for extracting information about plasticity in a $\mathrm{Zr}$-based $\mathrm{BMG}, \mathrm{Zr}_{41.2}$ $\mathrm{Ti}_{13.8} \mathrm{Cu}_{12.5} \mathrm{Ni}_{10} \mathrm{Be}_{22.5}{ }^{3}$ Tests were performed on micropillars of $0.3,1$, and $3 \mu \mathrm{m}$ diameters and on samples that were in the as-cast (AC), structurally relaxed (SR), and shot-peened (SP) states. These states correspond to 
extremely low and high free volumes (SR and SP states, respectively) and hence allow for a critical assessment as to how free volume affects the plasticity of BMGs. The experimental results show no significant differences either in strength or plastic flow characteristics (i.e., shear banding) with size of the specimen or its condition, despite the fact that the toughness of the BMG is markedly different in the three different conditions investigated. This result is because of the intrinsic limitations associated with uniaxial compression testing of materials that undergo inhomogeneous plasticity; namely, frictional contact at the platen-specimen surface initiating localized flow and shear bands and, subsequently, facilitation of relative sliding of one part of the specimen with respect to the other without ever reaching the critical condition for initiating fracture.

In this article, we examine whether the instrumented indentation tests can reveal any distinct features associated with plasticity in those three different conditions. Indentation methods offer many advantages, which include the following: (i) the mere requirement of relatively small material volumes for conducting a large number of tests; (ii) the relative ease with which they can be performed (a flat surface of rigidly held specimen is only needed); and (iii) the allowance for large plastic straining, mainly due to the constrained compressive stress state that imparts intrinsic stability to the deformation. In addition, hardness measurements can help to identify the extent of pressure sensitivity to plastic flow through the constraint factor, given by the ratio of hardness to yield strength. ${ }^{4-7}$ As a result, it has been often used in studying the mechanical behavior of metallic glasses.

\section{EXPERIMENTS}

A 3-mm-thick amorphous $\mathrm{Zr}_{41.2} \mathrm{Ti}_{13.8} \mathrm{Cu}_{12.5} \mathrm{Ni}_{10} \mathrm{Be}_{22.5}$ BMG plate $\left(T_{\mathrm{g}} \approx 625 \mathrm{~K}\right)$ in $\mathrm{AC}, \mathrm{SR}$, and SP conditions was investigated in this study. The as-cast BMG was annealed at $563 \mathrm{~K}$ for $12 \mathrm{~h}$ to induce structural relaxation. Structural characterization by means of differential scanning calorimetry and x-ray diffraction were performed on the relaxed material. Results of these and detailed analysis of free-volume reduction upon annealing can be found elsewhere. ${ }^{8,9}$ Shot peening was performed on polished surfaces of the as-cast BMG according to the AMS-S-13165 standard, using cast steel balls with $280 \mu \mathrm{m}$ mean diameter, resulting in a deformed layer thickness of $40 \mu \mathrm{m}$ and a residual compressive stress field of $\sim 150 \mu \mathrm{m} .{ }^{10}$ The surfaces of the bulk specimens were polished to a mirror finish before instrumented indentation was performed on a MTS NanoXP indenter (Eden Prairie, MN) at constant loading rates up to a maximum load of $500 \mathrm{mN}$ using cube corner and Berkovich diamond tips. In addition, in situ indentation experiments inside a Zeiss DSM962 scanning electron microscope
(SEM; Oberkochen, Germany) were performed using a microindenter driven by a stack piezo with a built-in displacement measurement (a detailed description of the instrument can be found in Ref. 11). The SEM images $(\sim 0.1$ frames per second) during the experiment were recorded in a video file and later synchronized with the load-displacement curve. Cross-sections of particular indents were fabricated by a focused-ion beam (FIB) technique using a Tescan Lyra instrument (Brno, Czech Republic). Shear band patterns around the indents and cross-sectional profiles were finally examined using a Leo 1530 FEG SEM (Carl Zeiss, Oberkochen, Germany).

\section{RESULTS}

\section{A. P-h curves}

Figure 1 shows representative load, $P$, versus displacement into the surface, $h$, curves obtained using a cube corner indenter at a constant loading rate of 1.67 $\mathrm{mN} / \mathrm{s}$. Pronounced displacement instabilities (i.e., "popins") are visible during loading. The magnitude of the pop-ins (i.e., pop-in length) linearly increases with $h$ as shown in Fig. 2(a). (For reasons of clarity, they are each averaged over $200 \mathrm{~nm}$ displacement intervals.) This linear relationship may simply be ascribed to the geometrical self-similarity of the sharp cube corner tip and the associated deformation field. ${ }^{12}$ No significant difference in the measured pop-in lengths between the AC and the SR material can be discerned (see also Table I). However, the BMG in the SP condition shows relatively large (and consequently fewer) pop-ins than the AC sample. The onset of pop-ins in the load-displacement curve is also more pronounced in this case.

Indentation at higher loading rates leads to a decrease of the pop-in lengths [compare Figs. 2(a) and 2(b)] as well as a decrease of the number of pop-ins. As the

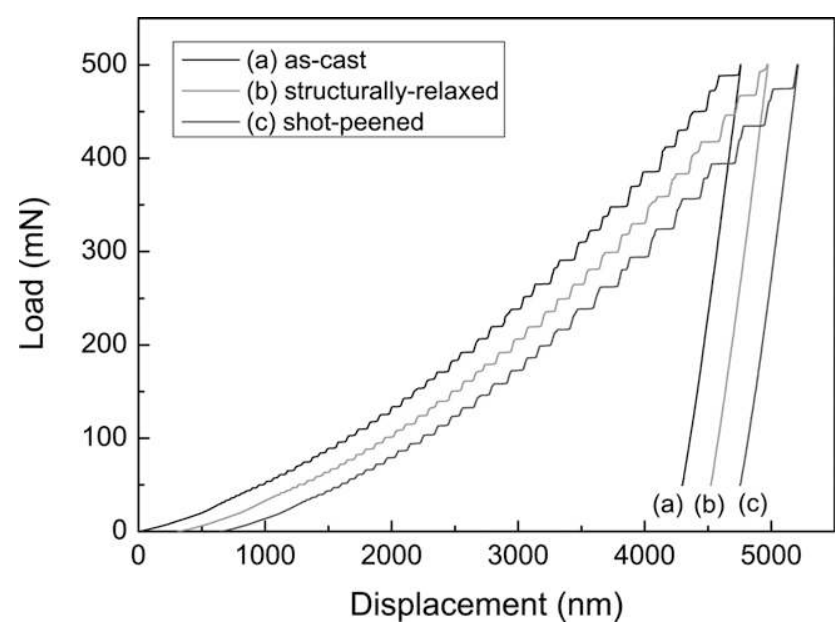

FIG. 1. Characteristic cube corner indentation curves for the differently treated Vit 1 samples at a loading rate of $1.67 \mathrm{mN} / \mathrm{s}$. The curves are shifted relative to each other for better visibility. 

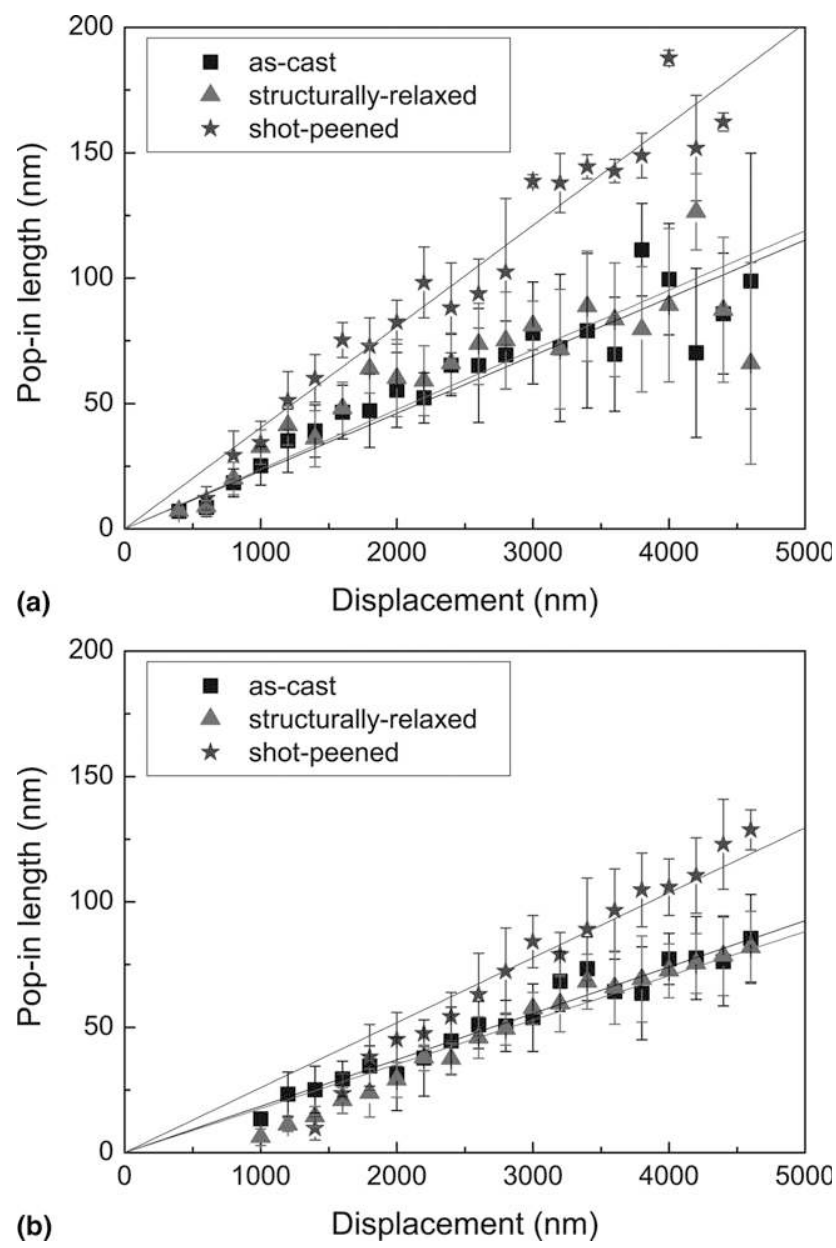

FIG. 2. Pop-in lengths for the differently treated Vit1 samples as a function of the displacement into the surface at a loading rate of (a) $1.67 \mathrm{mN} / \mathrm{s}$ and (b) $16.7 \mathrm{mN} / \mathrm{s}$, respectively. For reasons of clarity, the pop-in lengths are averaged over $200 \mathrm{~nm}$ displacement intervals; the solid lines are linear fits of the data.

TABLE I. Linear fits (in \%) of the pop-in lengths as a function of the indentation depths of the differently treated Vit1 samples for the two loading rates applied [see Figs. 2(a) and 2(b)].

\begin{tabular}{lcc}
\hline \hline \multicolumn{1}{c}{ Material condition } & $\begin{array}{c}\text { Low loading } \\
\text { rate }(1.67 \mathrm{mN} / \mathrm{s})\end{array}$ & $\begin{array}{c}\text { High loading } \\
\text { rate }(16.7 \mathrm{mN} / \mathrm{s})\end{array}$ \\
\hline As-cast & $2.30 \pm 0.08 \%$ & $1.85 \pm 0.03 \%$ \\
Structurally relaxed & $2.38 \pm 0.11 \%$ & $1.76 \pm 0.05 \%$ \\
Shot-peened & $4.04 \pm 0.09 \%$ & $2.59 \pm 0.08 \%$ \\
\hline \hline
\end{tabular}

loading rate is increased, the nature of the pop-ins changes from discrete, horizontal stair-step-like displacement bursts to more blurred fluctuations or ripples. These observations are consistent with earlier indentation studies (e.g., Refs. 12 and 13), which showed that the ratio of discrete plastic depth (i.e., individual popins) to total plastic depth decreases with increasing indentation loading rate. Furthermore, the apparent onset of pop-ins in the load-displacement curve is shifted to higher loads and indentation depths, respectively, with increasing loading rates. An increase of the applied loading rate from 1.67 to $16.7 \mathrm{mN} / \mathrm{s}$ shifts the apparent onset of pop-ins for each material's condition to higher values by approximately $40 \mathrm{mN}$ and $700 \mathrm{~nm}$, respectively.

\section{B. Shear band morphology}

Figure 3 shows the appearance of shear bands in the form of surface steps around the cube corner indents for the three different material states. There is no discernible difference between the AC and SR state; the top view images of the indents show a rather triangular pattern of the formed shear bands. However, the shear band appearance on the SP material is markedly different, and the shear bands form in a semicircular pattern. A slightly lower number of shear steps on the surface was observed on the SR sample compared with the AC sample, which is agreement with the lower shear band density on structurally relaxed and partially crystallized $\mathrm{Zr}_{52.5} \mathrm{Ti}_{5} \mathrm{Cu}_{17.9} \mathrm{Ni}_{14.6} \mathrm{Al}_{10}$ samples reported in Ref. 13. In contrast, significantly fewer shear bands are formed in the SP sample (see inclined images of Fig. 3). Keryvin ${ }^{14}$ showed that shear offsets on the surface are observed only when the indenter used induces strains large enough to reach the fully plastic regime. In this regard, the cube corner indenter, being extremely sharp, induces significantly large indentation strains, and hence leads to a fully plastic regime for all three conditions of the Zr-based BMG used in this study. Large differences in shear band number density and spacing among the AC, SR, and SP specimens can possibly be observed when blunt indenter tips are used. In fact, Murali and Ramamurty ${ }^{9}$ reported such difference between AC and SR samples tested with spherical indenters, with the SR sample showing significantly fewer shear bands. The authors attributed this embrittlement upon structural relaxation to the inability of the SR BMG to mitigate the crack-tip stress concentration through shear band-induced plasticity.

The cross-sections of the cube corner indents obtained by FIB-machining are shown in Fig. 4. The height of the shear offsets $(\Delta s)$ increases, whereas the angle of inclination with respect to the surface appears to decrease in the following sequence: AC, SR, and SP. In the SP case, the shear offsets have the appearance of wedge cracks.

Schuh et al. ${ }^{15}$ modeled the correlation between the pop-ins observed in the $P$ - $h$ curves and the shear offsets, which was verified by Moser et al. ${ }^{13}$ with the help of in situ indentation. Considering that the pop-in lengths of the AC and SR condition are similar (Fig. 2), the differences in the shear offset heights suggest a more prevalent reactivation of existing shear bands in the SR state. The observed large shear offset and low angle of inclination in the SP sample is associated with larger 


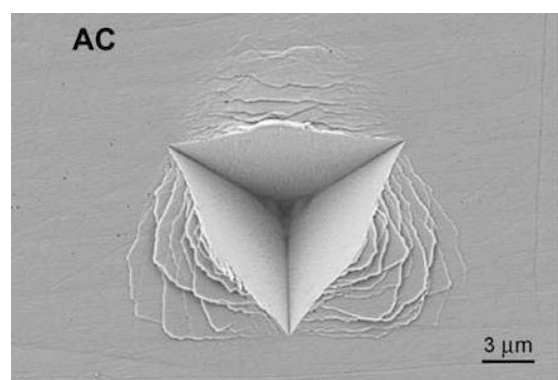

(a)

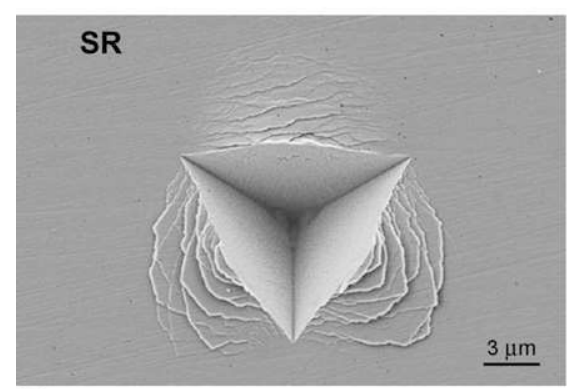

(b)

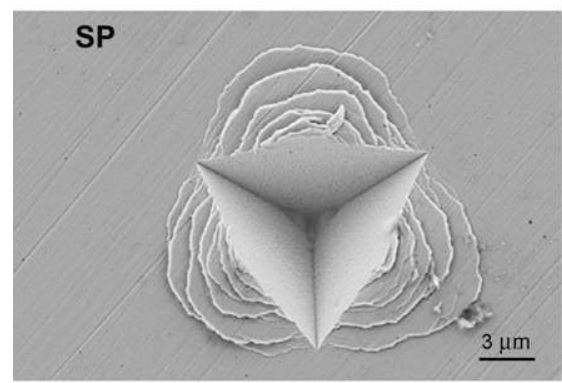

(c)
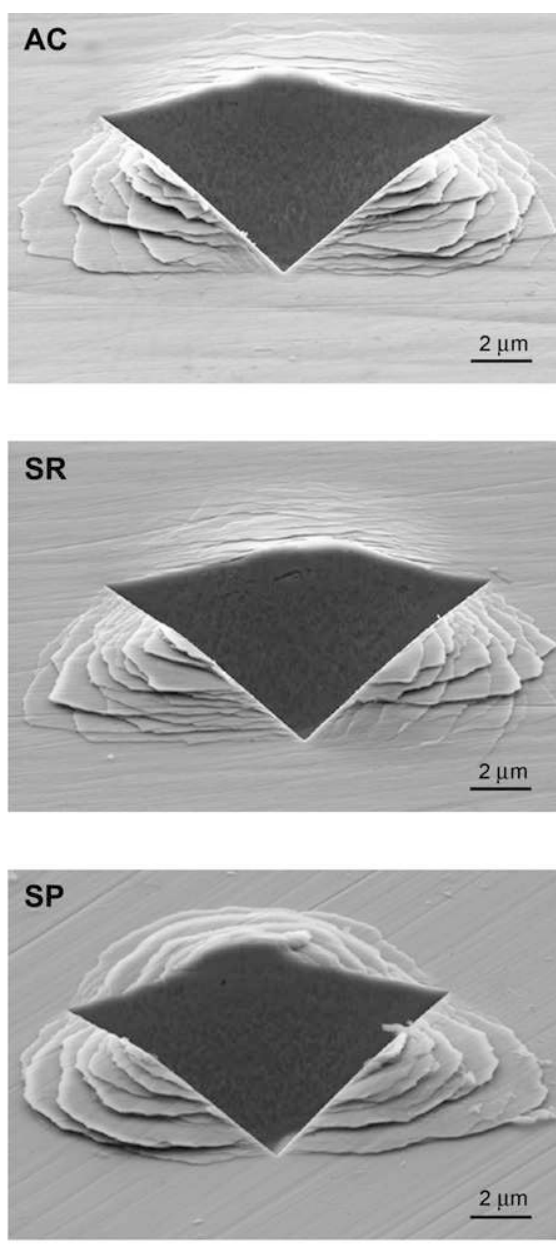

FIG. 3. SEM images [left, top view; right, inclined $\left(60^{\circ}\right)$ view] of cube corner indents (loading rate of $1.67 \mathrm{mN} / \mathrm{s}$ ) showing the appearance of shear bands in the form of surface steps for the (a) as-cast, (b) structurally relaxed, and (c) shot-peened material.

pop-in lengths and may be due to the biaxial compressive residual stresses that are present in the SP layer.

\section{Hardness}

The hardness (mean contact pressure) was calculated as $H=P_{\max } / A$, where $P_{\text {max }}$ is the maximum applied load and $A$ is the projected contact area of the cube corner indenter (including the pile-up, the area contribution of which is $\sim 20 \%$ ), determined via SEM image analysis. The values are listed in Table II. The biaxial compressive residual stress that exists in the peened layer of the SP sample, estimated to be $\sim 1.9 \mathrm{GPa}$ by Zhang et al., ${ }^{16}$ elevates the flow stress, and in turn enhances the measured hardness. ${ }^{17}$ Raghavan et al. ${ }^{10}$ who measured the hardness on the transverse section of the peened layer using a nanoindenter with a Berkovich tip, found the hardness values of the peened layer to be actually lower than the bulk hardness. This is because the biaxial residual stress is relaxed due to sectioning and the "actual" hardness is lower because of the deformation-induced soften- ing. ${ }^{18}$ However, it is well known that the Oliver and Pharr (OP) method $^{19}$ overestimates the hardness when there is substantial pile-up against the indenter due to plastic flow. Because the authors of Ref. 10 report significant pile-up in their cross-section indentations and have used the OP method, the hardness values reported by them cannot be used for further analysis herein.

Therefore, cube corner nanoindentation was performed on the sectioned SP layer in this work, and the mean contact pressure was accurately determined. The nanoindentation experiments were performed on a mirror-polished cross-section of the shot-peened layer $\sim 5 \mu \mathrm{m}$ from the surface using a Hysitron triboindenter (Minneapolis, MN) with a cube corner diamond tip (peak load of $5 \mathrm{mN}$, loading/unloading rate of $0.01 \mathrm{mN} / \mathrm{s}$, and peak load hold of $10 \mathrm{~s}$ ). Imaging of the indents was done by atomic force microscopy (AFM) using the same cube corner tip, and the projected area of the imprint was subsequently determined from these AFM images using the image analysis software Sigma scanpro 4.0 (Aspire Software International, Ashburn, VA). The average values 
of $H$, measured in this study, and the uniaxial compressive strengths, $\sigma_{\mathrm{y}}$, determined on $3-\mu \mathrm{m}$ micropillars in Ref. 3 are listed in Table II. It is seen that $H$ increases upon structural relaxation by $\sim 6 \%$, whereas it decreases by $\sim 14 \%$ upon shot peening (the AC values are used as the reference for comparison). Whereas $\sigma_{\mathrm{y}}$ can also be ranked in the same order as $H$, i.e., $\mathrm{SP}<\mathrm{AC}<\mathrm{SR}$, the relative change is small and within the experimental error.

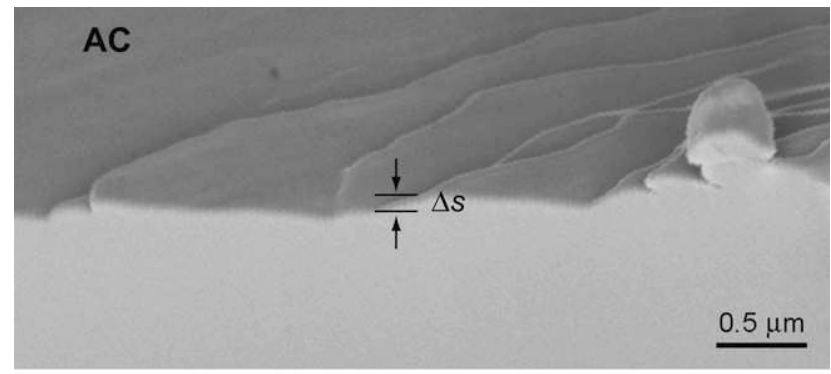

(a)

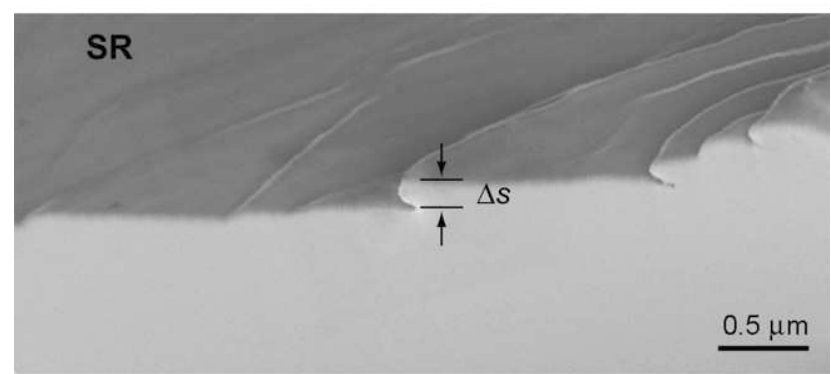

(b)

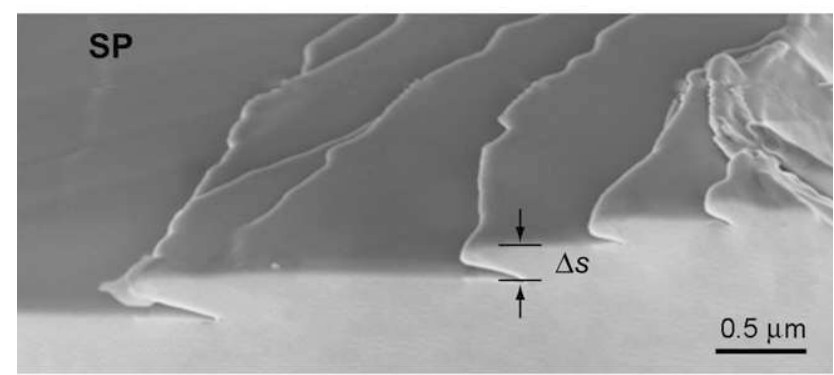

(c)

FIG. 4. FIB cut cross-sections of the shear offsets (i.e., surface steps next to the cube corner indent, which is at the right image border) for the (a) as-cast, (b) structurally relaxed, and (c) shoot-peened material. Schematically shown is also how the shear offset $\Delta s$ (with additional tilt correction) was determined.

\section{DISCUSSION}

\section{A. Pressure sensitivity}

During indentation, the matrix that surrounds the material underneath the indenter constrains the plastic flow. As a result, flow stress during indentation gets elevated significantly and is measured through the ratio of $H / \sigma_{y}$ in the fully plastic regime of indentation. Therefore, the ratio $H / \sigma_{\mathrm{y}}$ is referred to as the constraint factor, $C{ }^{6,7}$ In crystalline metals, $C \sim 2.6$ to 2.8 , whereas $C \geq 3$ for metallic glasses, a reflection of the sensitivity of their plastic flow to pressure. ${ }^{4,20}$ Furthermore, $C$ of BMGs as well as other amorphous materials like polymers increases with increasing temperature. ${ }^{5-7}$ The $C$ values, estimated using $H$ and $\sigma_{\mathrm{y}}$ and listed in Table II, can also be organized in the ascending order of $\mathrm{SP}<\mathrm{AC}<\mathrm{SR}$. Keryvin et al. ${ }^{6}$ have shown a one-to-one correspondence between $C$ and pressure sensitivity, and hence $C$ can be taken as the pressure sensitivity index. Consequently, the experimental results of this work show that pressure sensitivity of the BMG increases modestly upon structural relaxation, whereas it decreases considerably upon shot peening.

To further confirm these changes in pressure sensitivity due to changes in structural states, we have conducted conical indentations and examined the intersection angles, $\alpha$, between the two families of slip lines that emanate from the edge of contact of an axisymmetric indenter. If the material obeys the Tresca or the von Mises yield criterion, applicable for pressure-insensitive plasticity, the slip lines around a pressurized circular hole in a perfectly plastic solid under plane strain resemble that of logarithmic spirals, ${ }^{21}$ which follow the maximum shear directions with $\alpha=90^{\circ}$. For pressure-sensitive materials that obey Mohr-Coulomb yield criterion, the intersection angles are smaller, i.e., $\alpha<90^{\circ}$ and depend on the cone apex angle. ${ }^{22}$

The conical indentations on the AC and SR specimens were performed using a diamond cone tip (cone angle $120^{\circ}$, tip radius $0.2 \mathrm{~mm}$ ) that is normally used for Rockwell hardness measurements with an applied load of 980 N. Smaller indents were performed on the crosssection of the peened layer of the SP specimen using the MTS NanoXP indenter with a conical tip (cone angle $84.6^{\circ}$, tip radius $1 \mu \mathrm{m}$ ) and a maximum load of $100 \mathrm{mN}$.

TABLE II. Yield strength, $\sigma_{\mathrm{y}}$ (data obtained by conducting uniaxial compression tests on 3- $\mu \mathrm{m}$ micropillars, see Ref. 3 ), hardness, $H$, constraint factor $C\left(=H / \sigma_{\mathrm{y}}\right)$, and intersection angle $\alpha$ of the differently treated Vit1 samples. Data in parentheses indicate percentage change in property with respect to the as-cast state.

\begin{tabular}{lllr}
\hline \hline Material condition & \multicolumn{1}{c}{$\sigma_{\mathrm{y}}(\mathrm{MPa})$} & $H(\mathrm{GPa})$ & $C$ \\
\hline As-cast & $2079 \pm 52$ & $6.07 \pm 0.04$ & 2.92 \\
Structurally relaxed & $2137 \pm 110(+2.8 \%)$ & $6.45 \pm 0.05(+6.3 \%)$ & $3.02(+3.4 \%)$ \\
Shot-peened & $2030 \pm 49(-2.4 \%)$ & $5.22 \pm 0.21(-14 \%)$ & $2.57(-12 \%)$ \\
\hline \hline
\end{tabular}


The morphology around conical indents (i.e., the intersection angle of different families of shear bands) was examined by optical microscopy and SEM.

Figure 5 shows representative micrographs with families of shear bands intersecting each other, for the three structural states examined. The average $\alpha$ values

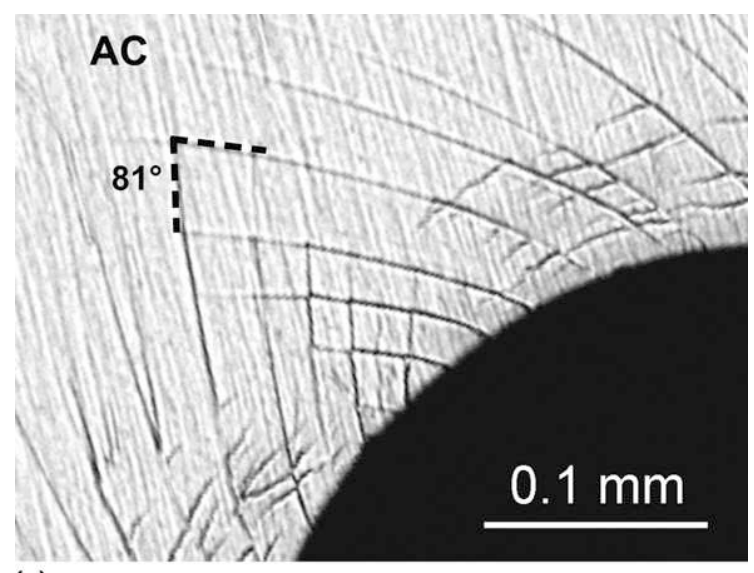

(a)

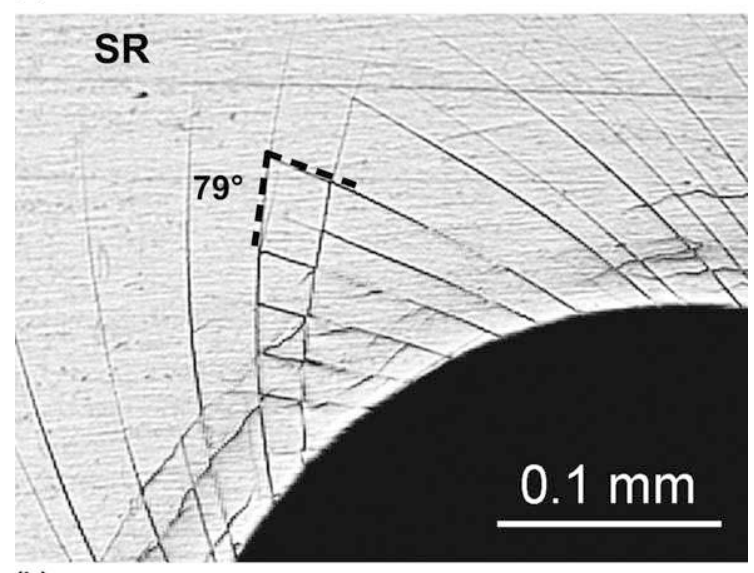

(b)

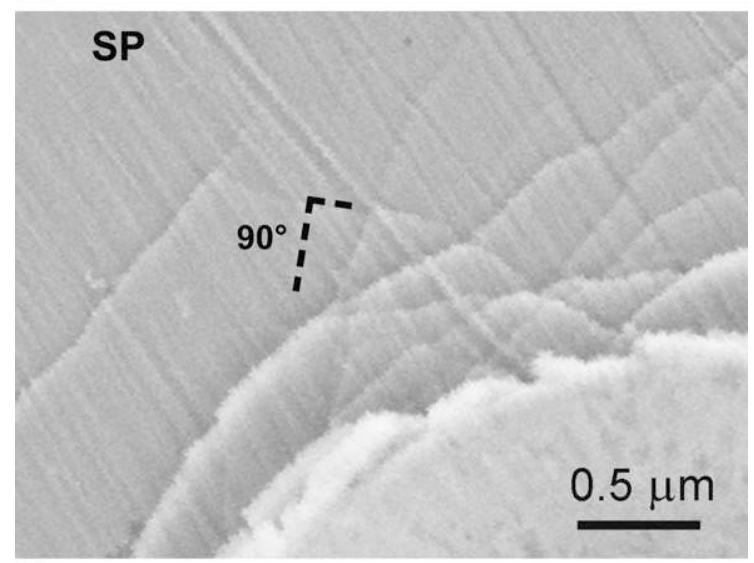

(c)

FIG. 5. Optical micrographs and SEM image, respectively, showing the intersecting slip lines (with the intersection angle $\alpha$ ) emanating from the edge of a conical indent, performed on the (a) as-cast and (b) structurally-relaxed material, and (c) on the cross-section of the shot-peened layer. measured from such micrographs are listed in Table II. For the AC condition, $\alpha=80.9^{\circ}$, close to that of $\sim 79^{\circ}$ reported by Patnaik et al. ${ }^{4}$ on the same alloy, which is representative of a material that obeys the Mohr-Coulomb yield criterion with a friction parameter of 0.19. Upon structural relaxation, $\alpha$ decreases marginally to $79.8^{\circ}$. This observation is in excellent agreement with the reduction in $\alpha$ (for similar temperature and time of annealing) upon structural relaxation reported by $\mathrm{Yu}$ et $\mathrm{al}^{23}$ The reduction in $\alpha$ suggests slightly enhanced pressure sensitivity with relaxation, which is consistent with the small increase in $C$.

The mechanistic origin for the enhanced $\sigma_{\mathrm{y}}$ and $H$ as well as pressure sensitivity upon structural relaxation can be understood as the following. The primary carriers of plasticity in amorphous materials are the shear transformation zones (STZs). ${ }^{24-26}$ Because STZ operation requires significant dilatation of the matrix that surrounds it, availability of free volume is critical for the STZs to occur in a given volume of material. Therefore, STZs occur preferentially in those regions that are less densely packed (i.e., have higher free-volume content) as relatively less dilatation of the matrix is required. It is well known that annealing leads to a reduction/annihilation of free volume, ${ }^{9}$ which in turn results in a densification of the BMG. ${ }^{27}$ This process makes the operation of STZs difficult (due to "higher atomic friction"), which results in an increased resistance to plastic deformation and therefore enhancement of yield strength and hardness of the BMG upon structural relaxation. This effect is consistent with other studies that report an enhancement in hardness upon structural relaxation. ${ }^{28,29}$

Turning attention to the SP condition, the measured $\alpha$ is $\sim 90^{\circ}$, indicating that the $\mathrm{BMG}$ in this structural state obeys Tresca or von Mises yield criteria, which rely only on the deviatoric component of the stress tensor, as in crystalline metals. In addition, the $C=2.57$ obtained for the SP case is close to that obtained in crystalline metals, ${ }^{30}$ confirming that the BMG indeed becomes pressure-insensitive upon exposure to severe plastic deformation. The free-volume model, proposed by Spaepen $^{31}$ for plastic flow in metallic glasses, implies the creation of free volume during the deformation, which in turn leads to local reduction in viscosity. Such freevolume enhancements were confirmed experimentally in Refs. 18 and 32 among others. However, the excess free volume generated during plastic deformation is not stable, ${ }^{33}$ and it coalesces to form nanovoids once the externally applied stress is removed, as experimentally verified by Li et al. ${ }^{34}$ and Flores et al. ${ }^{35}$ Nanoindentation of ball-indented plastic regions showed that these nanovoids act as sites for the initiation of shear bands. ${ }^{18}$ Because the shot-peening process involves repeated indentations that induce successive plastic strains in the surface layers, it is likely that the effective free volume 
in those layers increases considerably. Converse to that seen in SR samples, where reduction in free volume enhances hardness and also pressure sensitivity, increase in free volume due to shot peening decreases the flow stress and hardness. It is interesting to note that the freevolume increase is so significant that pressure sensitivity completely vanishes, which implies that in this case the operation of STZs does not involve any dilatational component.

\section{B. Advantages of the indentation method}

The results presented in the preceding section clearly show that indentation method exposes more critical information about plasticity in amorphous alloys than uniaxial compression. This is because hardness is more sensitive to the structural state of the BMG than the uniaxial compressive yield strength. Possible reasons for this are presented here. First, it is essential to recognize that plastic deformation in amorphous alloys involves both homogeneous and inhomogeneous strains. ${ }^{25}$ The former occurs due to STZ operation throughout the solid and contributes $100 \%$ to plasticity in the supercooled liquid regime (i.e., at temperatures above the glass transition temperature, $T_{\mathrm{g}}$ ). At temperatures lower than $T_{\mathrm{g}}$, in addition to STZs, shear localization occurs at high stresses along the planes of maximum shear, leading to inhomogeneous plasticity. The onset of shear banding often manifests as the yield stress in uniaxial compression, although a considerable amount of homogeneous deformation through STZ operation may take place before that. (Note that shear bands and STZs are interconnected, with the former linking several STZs. However, the mechanistic connection between STZs and shear bands has not been fully established yet.) Thus, uniaxial tests reflect only the shear banding activity unless careful experiments such as acoustic emission spectroscopy are performed to "pick" STZ activity. ${ }^{36}$

On the other hand, indentation tests are more sensitive to the homogeneous plasticity. This is amply demonstrated by the following observation. For up to $\sim 1 \mu \mathrm{m}$ of cube corner indentation, no discrete displacement bursts, which are associated with the shear bands, are observed in the $P$ - $h$ curves [Fig. 2(a)]. Because cube corner tips (or for that matter, any of the sharp indenters such as Vickers, Berkovich, or conical) are affine in nature, there will be plastic strain, which is independent of $h$. Thus, one can deduce that the plastic strain during the early stages of indentation of amorphous alloys arises due to homogeneous plasticity. This finding is also supported by the fact that at lower indentation strains, indentation impressions are present although no shear band activity around the indent can be seen. A similar observation was made in a series of in situ SEM tensile tests on amorphous metallic wires, where homogeneous plastic deformation before shear band activity was observed. ${ }^{37}$

At large depth of penetration, deformation through shear bands occurs, amply reflected by the pop-ins in the $P$ - $h$ curves in Fig. 1 as well as by the observation of indents in Fig. 3. This observation suggests that whereas the plasticity that occurs through inhomogeneous flow (i.e., shear banding) during sharp indentation may be similar, plasticity that occurs through STZ operation is affected because of the large hydrostatic compressive stress that is present underneath the indenter. This hydrostatic stress makes the STZ operation difficult, which in turn elevates the hardness. Thus, critical examination of indentation tests on amorphous alloys can reveal vital information about the underlying physics of deformation.

\section{SUMMARY}

Detailed instrumented indentation was carried out on a Zr-based BMG with varying amounts of free volume. The experimental observations show significant differences in hardness and slight changes in the shear band morphology around the indents depending on the structural state of the BMG, while its yield strength remains roughly invariant. The different behavior can be rationalized in terms of a free-volume dependent pressure sensitivity, quantified by the constraint factor (i.e., hardness-to-yield-strength ratio) and the intersection angle of emanating shear bands at the edge of conical indents, respectively. Whereas structural relaxation leads to an enhanced pressure sensitivity compared with the as-cast state, pre-deformation by means of shot peening results in a pressure-insensitive deformation response of the BMG. Microscopic origin of this behavior is the ease of STZ operation, which requires local dilatation and hence is prone to be activated more easily in less dense glassy structures. As a consequence, it would be interesting to study whether the change in pressure sensitivity with varying amounts of free volume is also reflected in a change of the STZ volume and activation volume, respectively.

\section{ACKNOWLEDGMENT}

This work was supported by the Swiss National Science Foundation (Grant No. 200020-120258).

\section{REFERENCES}

1. R.D. Conner, W.L. Johnson, N.E. Paton, and W.D. Nix: Shear bands and cracking of metallic glass plates in bending. J. Appl. Phys. 94, 904 (2003).

2. A. Castellero, D.I. Uhlenhaut, B. Moser, and J.F. Löffler: Critical Poisson ratio for room-temperature embrittlement of amorphous $\mathrm{Mg}_{85} \mathrm{Cu}_{5} \mathrm{Y}_{10}$. Philos. Mag. Lett. 87, 383 (2007). 
3. A. Dubach, R. Raghavan, J.F. Löffler, J. Michler, and U. Ramamurty: Micropillar compression studies on a bulk metallic glass in different structural states. Scr. Mater. 60, 567 (2009).

4. M.N.M. Patnaik, R. Narasimhan, and U. Ramamurty: Spherical indentation response of metallic glasses. Acta Mater. 52, 3335 (2004).

5. K. Eswar Prasad, R. Raghavan, and U. Ramamurty: Temperature dependence of pressure sensitivity in a metallic glass. Scr. Mater. 57, 121 (2007)

6. V. Keryvin, K. Eswar Prasad, Y. Gueguen, J-C. Sangleboeuf, and U. Ramamurty: Temperature dependence of mechanical properties and pressure sensitivity in metallic glasses below glass transition. Philos. Mag. 88, 1773 (2008).

7. K. Eswar Prasad, V. Keryvin, and U. Ramamurty: Pressure sensitive flow and constraint factor in amorphous materials below glass transition. J. Mater. Res. 24, 890 (2009).

8. R. Raghavan, P. Murali, and U. Ramamurty: Ductile to brittle transition in the $\mathrm{Zr}_{41.2} \mathrm{Ti}_{13.75} \mathrm{Cu}_{12.5} \mathrm{Ni}_{10} \mathrm{Be}_{22.5}$ bulk metallic glass. Intermatallics 14, 1051 (2006).

9. P. Murali and U. Ramamurty: Embrittlement of a bulk metallic glass due to sub- $T_{\mathrm{g}}$ annealing. Acta Mater. 53, 1467 (2005).

10. R. Raghavan, R. Ayer, H.W. Jin, C.N. Marzinsky, and U. Ramamurty: Effect of shot peening on the fatigue life of a $\mathrm{Zr}$ based bulk metallic glass. Scr. Mater. 59, 167 (2008).

11. R. Rabe, J.M. Breguet, P. Schwaller, S. Stauss, F.J. Huag, J. Patscheider, and J. Michler: Observation of fracture and plastic deformation during indentation and scratching inside the scanning electron microscope. Thin Solid Films 469, 206 (2004).

12. C.A. Schuh and T.G. Nieh: A nanoindentation study of serrated flow in bulk metallic glasses. Acta Mater. 51, 87 (2003).

13. B. Moser, J.F. Löffler, and J. Michler: Discrete deformation in amorphous metals: An in situ SEM indentation study. Philos. Mag. 86, 5715 (2006).

14. V. Keryvin: Indentation of bulk metallic glasses: Relationships between shear bands observed around the prints and hardness. Acta Mater. 55, 2565 (2007).

15. C.A. Schuh, A.C. Lund, and T.G. Nieh: New regime of homogeneous flow in the deformation map of metallic glasses: Elevated temperature nanoindentation experiments and mechanistic modeling. Acta Mater. 52, 5879 (2004).

16. Y. Zhang, W.H. Wang, and A.L. Greer: Making metallic glasses plastic by control of residual stress. Nat. Mater. 5, 857 (2006).

17. L.Y. Chen, Q. Ge, S. Qu, and J.Z. Jiang: Stress-induced softening and hardening in a bulk metallic glass. Scr. Mater. 59, 1210 (2008).

18. R. Bhowmick, R. Raghavan, K. Chattopadhyay, and U. Ramamurty: Plastic flow softening in a bulk metallic glass. Acta Mater. 54, 4221 (2006).

19. W.C. Oliver and G.M. Pharr: An improved technique for determining hardness and elastic modulus using load and displacement sensing indentation experiments. J. Mater. Res. 7, 1564 (1992).

20. U. Ramamurty, S. Jana, Y. Kawamura, and K. Chattopadhyay: Hardness and plastic deformation in a bulk metallic glass. Acta Mater. 53, 705 (2005).
21. L.M. Kachanov: Fundamentals of the Theory of Plasticity (Mir Publishers, Moscow, Russia, 1974), p. 22.

22. V. Keryvin, R. Crosnier, R. Laniel, V.H. Hoang, and J-C. Sangleboeuf: Indentation and scratching mechanisms of a ZrCuAlNi bulk metallic glass. J. Phys. D: Appl. Phys. 41, 074029 (2008)

23. G.S. Yu, J.G. Lin, M. Mo, X.F. Wang, F.H. Wang, and C.E. Wen: Effect of relaxation on pressure sensitivity index in a Zr-based metallic glass. Mater. Sci. Eng., A 460, 58 (2007).

24. A.S. Argon: Plastic deformation in metallic glass. Acta Metall. 27, 47 (1979).

25. C.A. Schuh, T.C. Hufnagel, and U. Ramammurty: Mechanical behavior of amorphous alloys. Acta Mater. 55, 4067 (2007).

26. A. Dubach, F.H. Dalla Torre, and J.F. Löffler: Constitutive model for inhomogeneous flow in bulk metallic glasses. Acta Mater. 57, 881 (2009).

27. D.I. Uhlenhaut, F.H. Dalla Torre, A. Castellero, C.A.P. Gomez, N. Djourelov, G. Krauss, B. Schmitt, B. Patterson, and J.F. Löffler: Structural analysis of rapidly solidified $\mathrm{Mg}-\mathrm{Cu}-\mathrm{Y}$ glasses during room-temperature embrittlement. Philos. Mag. 89, 233 (2009).

28. S. Xie and E.P. George: Hardness and shear band evolution in bulk metallic glasses after plastic deformation and annealing. Acta Mater. 56, 5202 (2008).

29. B-G. Yoo, K-W. Park, J-C. Lee, U. Ramamurty, and J-I. Jang: Role of free volume in strain softening of as-cast and annealed bulk metallic glass. J. Mater. Res. 24, 1405 (2009).

30. M. Dao, N. Chollacoop, K.J. Van Vliet, T.A. Venkatesh, and S. Suresh: Computational modeling of the forward and reverse problems in instrumented sharp indentation. Acta Mater. 49, 3899 (2001).

31. F. Spaepen: A microscopic mechanism for steady state inhomogeneous flow in metallic glasses. Acta Metall. 25, 407 (1977).

32. K. Hajlaoui, T. Benameur, G. Vaughan, and A.R. Yavari: Thermal expansion and indentation-induced free volume in $\mathrm{Zr}$-based metallic glasses measured by real-time diffraction using synchrotron radiation. Scr. Mater. 51, 843 (2004).

33. W.J. Wright, T.C. Hufnagel, and W.D. Nix: Free volume coalescence and void formation in shear bands in metallic glass. J. Appl. Phys. 93, 1432 (2003).

34. J. Li, Z.L. Wang, and T.C. Hufnagel: Characterization of nanometer-scale defects in metallic glasses by quantitative high-resolution transmission electron microscopy. Phys. Rev. B: Condens. Matter 65, 144201 (2002).

35. K.M. Flores, E. Sherer, A. Bharathula, H. Chen, and Y.C. Jean: Sub-nanometer open volume regions in a bulk metallic glass investigated by positron annihilation. Acta Mater. 55, 3403 (2007).

36. A.Y. Vinogradov and V.A. Khonik: Kinetics of shear banding in a bulk metallic glass monitored by acoustic emission measurements. Philos. Mag. 84, 2147 (2004).

37. B. Zberg, E.R. Arata, P.J. Uggowitzer, and J.F. Löffler: Tensile properties of glassy $\mathrm{MgZnCa}$ wires and reliability analysis using Weibull statistics. Acta Mater. 57, 3223 (2009). 